\title{
Screening for Cancer-Related Neuropathic Pain in the Oncology Outpatient Setting in the United Kingdom
}

\author{
Alison Birtle ${ }^{1, *}$, Susan Davidson ${ }^{2}$, Gary Atkinson ${ }^{3}$ and Chantal van Litsenburg ${ }^{4}$
}

\author{
${ }^{I}$ Rosemere Cancer Centre, Lancashire Teaching Hospitals NHS Foundation Trust, Royal Preston Hospital, Sharoe \\ Green Lane North, Preston, UK; ${ }^{2}$ The Christie NHS Foundation Trust, Wilmslow Road, Manchester, UK; ${ }^{3}$ Pfizer Ltd, \\ Walton Oaks, UK; ${ }^{4}$ Pfizer bv, Capelle a/d IJssel, The Netherlands
}

\begin{abstract}
This article reports the outcomes of a sub-analysis of United Kingdom (UK) data collected during a noninterventional, cross-sectional study conducted in five European countries. The primary aim was to estimate the prevalence of cancer-related neuropathic pain (CRNP) in an outpatient sample of adult cancer patients visiting oncology clinics in the UK for standard care. Secondary aims were to report the nature and characteristics of the cancer and the pain in the patients with CRNP. This sub-analysis also assessed the usefulness of the PainDETECT screening tool as an aid for physicians in identifying the neuropathic component of cancer-related pain in daily practice. Based on physicians' clinical judgment before reviewing the scores on the PainDETECT tool, the estimated number of outpatients with cancer experiencing chronic pain and considered to have CRNP was 104 of 195 patients $(53.3 \%$; $95 \%$ confidence interval [CI]: $46.3 \%$ $60.3 \%$ ). After reviewing patients' scores on the tool, the estimate was 90 of 195 patients (46.2\%; $95 \%$ CI: $39.2 \%-53.2 \%$ ). Physicians changed from a positive (yes) to a negative (no) diagnosis of CRNP for 16 of 127 patients who had a low PainDETECT end score $(<13$; indicating that neuropathic pain was unlikely). Of the 11 physicians who completed the usefulness of PainDETECT survey, eight indicated that they would use the questionnaire in future for at least some of their patients, although they also indicated that in the majority of cases $(63 \%)$, the PainDETECT tool did not help them evaluate whether a patient had CRNP. Because of missing data arising from missing or incomplete survey responses, however, these data should be interpreted with caution, and further studies are required to assess the usefulness of this tool.
\end{abstract}

Keywords: Clinical oncology, neuropathic pain, outpatients, questionnaire, PainDECTECT.

\section{INTRODUCTION}

Over 300,000 patients are diagnosed with cancer each year in the United Kingdom (UK) [1]. The European agestandardised rates based on estimated 2009 incidence rates in the UK were 429 cases per 100,000 person-years for men and 372 cases per 100,000 person-years for women [2]. Up to $53 \%$ of cancer patients experience cancer-related pain, irrespective of disease stage or tumour type [3]. Of those experiencing pain, over one-third grade their pain as moderate or severe [3], yet pain in patients with cancer frequently remains undertreated [4-6]. Undertreatment of pain is multifactorial and can result from under-reporting of the pain by the patient. It may also reflect lack of experience in detection or management of chronic pain by the treating oncologist $[4,5]$.

Effective management of pain is partly dependent on correctly identifying the source and type of pain, which can be either nociceptive (somatic or visceral) or neuropathic (somatosensory). Individuals can also experience nociceptive and neuropathic pain simultaneously [7,8]. Patients with cancer represent one of the most challenging populations with regard to pain management. Effective pain management

\footnotetext{
*Address correspondence to this author at the Rosemere Cancer Centre, Royal Preston Hospital, Sharoe Green Lane North, Preston, PR2 9HT, UK; Tel: +44 (0)1772 524762; Fax: +44 (0)1772 522178;

E-mail: Alison.Birtle@1thtr.nhs.uk
}

within this population is complicated by the stage of disease, the type of cancer, and the use of concomitant cancer treatments, as well as the nature of the pain [9, 10]. Like neuropathic pain in general, neuropathic pain in patients with cancer has been identified as more difficult to manage and can be experienced more intensely than pain without a neuropathic component $[11,12]$. Additionally, for patients with neuropathic cancer pain, it is important to distinguish between neuropathic pain that is caused directly by the tumor and neuropathic pain that is caused by the cancer treatment (e.g., chemotherapy-induced painful peripheral neuropathy) [13]. Consequently, improving the understanding of the characteristics and nature of pain experienced by patients with cancer may lead to more effective pain management within this population.

One factor contributing to the undertreatment of pain in cancer patients is the failure to identify neuropathic pain components that may require additional pharmacologic treatment [14]. Based on data from international studies conducted in a variety of inpatient and outpatient settings, Bennett et al. [14] estimated that up to $39 \%$ of patients with cancer may experience pain with a neuropathic component. There are several screening or assessment tools designed to identify neuropathic pain, including the PainDETECT questionnaire, which was originally developed to identify neuropathic pain in patients with chronic lower back pain [15]. PainDETECT has since been validated, and is in common 
use, to identify neuropathic pain in other conditions, for example fibromyalgia [16] and painful diabetic neuropathy and peripheral neuropathy [17].

A non-interventional, cross-sectional study conducted in five European countries, including the UK, estimated that $32.6 \%$ of outpatients experiencing chronic pain experienced cancer-related neuropathic pain (CRNP) [18]. This is consistent with estimates from international studies conducted in a variety of inpatient and outpatient settings that up to $39 \%$ of patients with cancer may experience pain with a neuropathic component [14]. The current sub-analysis is based on the UK data collected during the five-country European study. Identifying the scale of CRNP on a country-level is important and may assist the individual countries strengthen their care systems and the management of cancer pain. The primary objective of this subanalysis was to estimate the prevalence of CRNP in an outpatient sample of adult cancer patients visiting oncology clinics for standard care. Secondary aims included reporting the nature and characteristics of the cancer and the pain in the UK subpopulation of patients with CRNP and assessing the physicians self-reported usefulness of the PainDETECT screening tool [15] as an aid to physicians in identifying the neuropathic component of cancerrelated pain in daily practice.

\section{MATERIALS AND METHODOLOGY}

\section{Study Design}

The data reported here were collected from 12 physicians from eight UK centres as part of an observational, noninterventional, cross-sectional, multicentre study. The main study was conducted between 23 August 2010 and 22 July 2011 in outpatient oncology clinics in Denmark, Germany, Greece, Spain, and the UK. Data relating to diagnosis and disease management were collected retrospectively. The protocols were reviewed and approved by Institutional Review Boards/Independent Ethics Committees at participating centres. The Central Multicentre Research Ethics Committee Reference Number was 10/H1011/50.

\section{Patients}

All patients had chronic cancer-related pain (defined as pain most days of the week for $\geq 3$ months). They were adults aged at least 18 years and identified by their clinicians as having chronic cancer-related pain immediately prior to their routine follow-up consultation in the oncology outpatient clinic. Patients with any solid tumour malignancy and at any stage of disease (metastatic, locally advanced, or nonmetastatic) were screened for eligibility. Patients with chronic pain unrelated to cancer were excluded from the study. All patients provided informed consent and were willing to participate in the study.

\section{Study Enrolment and Procedures}

Patients were screened and identified for eligibility during routine consecutive consultations with the physician during the enrolment period. The study was planned to include every patient in a screening log at the time that such patient was considered for participation, in order to minimise potential recruitment bias. Patients with cancer-related chronic pain completed the neuropathic pain screening tool (PainDETECT) [15]. This tool was used to identify patients with
CRNP based on the physicians' clinical judgments before and after reviewing the PainDETECT scores. The CRNP population was defined based on physician opinion after consideration of the PainDETECT scores.

The PainDETECT tool is a self-report questionnaire that allows descriptive reporting of the characteristics, intensity, and pattern of pain. It includes 0 -to-10 rating scales in relation to three preliminary questions, followed by a series of seven questions with a 0 to 5 rating scale to establish the nature of the pain. The sum of scores from these seven questions (maximum subscore $=35$ ), together with a 3-point "radiating pain" score, are totalled (maximum total score $=38$ ), and used to establish the likelihood of the patient's having neuropathic pain (end scores of $>18=$ likely; $13-18=$ possible; $<13=$ unlikely) [15].

The CRNP population was assessed for additional characteristics, and pharmacologic management of the pain and the cancer, via case report forms (CRFs) completed by the patients and their physicians, as well as other self-report questionnaires.

After all patients completed the study, physicians completed an investigator-specific CRF relating to the PainDETECT questionnaire. Physicians who completed this CRF were defined as the "surveyed physicians" population.

\section{Outcome Measures}

\section{CRNP Prevalence}

CRNP prevalence, calculated as the number of patients with CRNP compared with the total number of patients with chronic pain, was estimated before and after physicians' assessment of PainDETECT scores.

\section{Chronic Pain Prevalence}

The prevalence of chronic pain was calculated using a point estimate of the number of patients experiencing pain compared with the total number of patients with recorded screening data.

\section{Nature, Treatment, and Characteristics of CRNP and Cancer}

The characteristics, history, and treatment of pain were assessed via investigator and patient CRFs and via the Modified Brief Pain Inventory Short Form (m-BPI-sf) [19]. The characteristics, history, and treatment of cancer were also assessed via investigator and patient CRFs.

\section{Health-Related Quality of Life (QoL) in CRNP Patients}

QoL was assessed via the EQ-5D Health Questionnaire (EuroQoL) [20] and the Sheehan Disability Scale (SDS) [21].

\section{Physicians' Assessment of the PainDETECT Question- naire}

The usefulness of the PainDETECT screening tool was evaluated in the surveyed physicians population via the physicians self-reported usefulness which was documented in the investigator-specific CRF.

\section{Statistical Analysis}

\section{Sample Size}

The study plan called for 200 patients to be enrolled in the UK to achieve an estimated precision of $\pm 7 \%(95 \% \mathrm{CI}$ for the prevalence of CRNP). However, due to difficulties 
with investigator enrolment, the total number of patients enrolled fell just short of this number $(n=195)$. This shortfall had little impact on the estimated precision.

\section{Data Analysis}

Discrete endpoints were summarised using frequency and percentage calculations for each response category. Missing data were excluded from the percentage calculations. Continuous endpoints were summarised using descriptive statistics.

\section{RESULTS}

\section{Patient and Surveyed Physicians' Populations}

Of 195 patients, $187(95.9 \%)$ completed all or part of the survey. Four patients $(2.1 \%)$ withdrew during the screening phase, four $(2.1 \%)$ were no longer willing to participate in the study, and data were missing (either CRF page or final status) for four $(2.1 \%)$ patients. A total of 12 physicians took part in the study, 11 of whom completed some or all of the investigator-specific CRF (surveyed physician population).

\section{Patient Demographics}

Of the 193 patients with properly recorded demographic data, over half of the patients $(57.5 \%)$ were female. Male patients were older (ages ranged from 31 to 87 years), on average, than the female patients (ages ranged from 20 to 84 years), and $75 \%$ of the men were aged 65 years and older, in comparison with $36 \%$ of the women (Table 1).

\section{Study Endpoints \\ CRNP}

Fewer patients (90 of 195) were considered to have CRNP in the opinion of the evaluating physician after reviewing scores on the PainDETECT questionnaire $(46.2 \%$; 95\% CI: $39.2 \%-53.2 \%$ ) than the number considered to have CRNP before reviewing these scores (104 of 195; 53.3\%; 95\% CI: 46.3\%-60.3\%; Fig. 1).

\section{Prevalence of Chronic Pain}

Data recorded on the screening log during the enrolment period were used to identify patients with chronic pain. Data from physicians invited to take part in the survey who did not record the number of patients screened were not included in this analysis. Consequently, the number of patients meeting the criteria for chronic pain included in the chronic pain calculation differs from the number of patients with chronic pain identified as the "all patient" population in the CRNP calculation. Of $2825 \mathrm{UK}$ patients with screening data, 162 met the criteria for chronic pain and were enrolled in the study, and 92 patients met the criteria and declined participation. ; all 254 patients were included in the calculation of the prevalence of chronic pain and constitute the numerator The prevalence point estimate of chronic pain was therefore calculated as follows:

$$
(162+92) \times 100 / 2825=9.0 \%
$$

\footnotetext{
${ }^{1}$ Patients who did not meet the criteria for chronic pain due to use of pain medications were not included in the chronic pain calculation (as these data were not always reliably recorded). The chronic pain calculation is, therefore, a conservative estimate.
}

Nature and Treatment of Neuropathic Pain in Patients with CRNP

In the physicians' assessment of the type of chronic cancer-related pain, 26 of the 90 patients in the CRNP population $(28.9 \%)$ were considered to experience pure neuropathic-type pain, and $36(40.0 \%)$ were considered to have mixed cancer-related pain with a neuropathic component. Data were missing for $28(31.1 \%)$ patients.

Of the CRNP population, three patients had experienced CRNP for $<3$ months $(3.3 \%) ; 30(33.3 \%)$ had had CRNP for 3-6 months; 11 (12.2\%) had had CRNP for 7-12 months, 15 (16.7\%) for 13 months to 3 years, and five $(5.6 \%)$ for $>3$ years. Data were missing for $26(28.9 \%)$ patients.

In the opinion of the physicians, the CRNP was due to the tumour itself in 48 of 90 cases $(53.3 \%)$, and due to cancer treatment in 24 cases $(26.7 \%)$. Chemotherapy and radiotherapy were each considered responsible for the CRNP in nine cases $(10.0 \%)$, and surgery was considered responsible in 12 cases $(13.3 \%)$. Non-opioid analgesics and/or strong opioids were the most commonly prescribed therapeutic treatments for neuropathic pain in the CRNP population (Table 2).

\section{Pain-Related Characteristics of CRNP Patients}

Mean subscale scores on the m-BPI-sf were 5.5 (95\% CI: 4.7-6.2; $\mathrm{n}=65)$ for "Interference" and 4.6 (95\% CI: 4.0-5.1; $\mathrm{n}=66$ ) for "Severity," based on numerical rating scales of 0 (no pain) to 10 ("pain as bad as you can imagine").

Patient-assessed pain scores derived from the preliminary PainDETECT questions were higher, on average, for the CRNP population than for the "all participant" group (Table 3). No statistical comparisons were performed between these two populations.

\section{Characteristics of Cancer and Treatment in the CRNP Population}

\section{Types and Characteristics of Cancer}

In the 90-patient CRNP population, breast cancer was the most common primary diagnosis $(n=20 ; 22.2 \%)$. Excluding the "other" category, the next most commonly recorded cancer type was prostate cancer $(n=6 ; 6.7 \%)$ (Table 4). Thirtyeight patients $(42.2 \%)$ had loco-regional progression of the cancer, $22(24.4 \%)$ did not, and data were missing for 30 $(33.3 \%)$ patients. Of the 90 patients, $44.4 \%$ of the CRNP population had metastatic disease at one or more sites (range of number of sites $=0-7$ ). Data were missing for 42 patients $(46.7 \%)$. Bone was the most common metastatic site (Table 4).

Summary of Therapeutic Management of Cancer and Surgical Treatment

At the time of the study, approximately half of the CRNP population had received prior or ongoing chemotherapy $(53.3 \%)$ and/or radiotherapy treatment $(55.6 \%)$. Patients could have received more than one type of therapeutic or surgical treatment for cancer.

For 24 of the 90 patients in the CRNP population (26.7\%), the surgery CRF was missing. The type of cancer treatment (neoadjuvant [i.e., cancer treatment prior to 
Table 1. Demographic Characteristics of Patient Populations

\begin{tabular}{|c|c|c|c|c|}
\hline & Male & Female & $\begin{array}{l}\text { Patients for Whom Sex } \\
\text { Was Not Recorded }\end{array}$ & Total \\
\hline All patients ${ }^{\mathrm{a}}, \mathrm{n}$ & 81 & 111 & 1 & 193 \\
\hline \multicolumn{5}{|l|}{ Age, years, n (\%) } \\
\hline $18-44$ & $3(3.7)$ & $15(13.5)$ & 0 & $18(9.3)$ \\
\hline Mean (SD) & $68.9(10.7)$ & $59.6(12.1)$ & $48.0(0)$ & $63.4(12.4)$ \\
\hline Patients with CRNP, $n$ & 31 & 57 & 1 & 89 \\
\hline \multicolumn{5}{|l|}{ Age, years, n (\%) } \\
\hline Mean (SD) & $67.3(10.1)$ & $56.6(12.4)$ & $48.0(0)$ & $60.2(12.7)$ \\
\hline
\end{tabular}

ancorrect date of birth was recorded for 2 patients in the "all patient" population and excluded from the demographic summary. CRNP, cancer-related neuropathic pain.

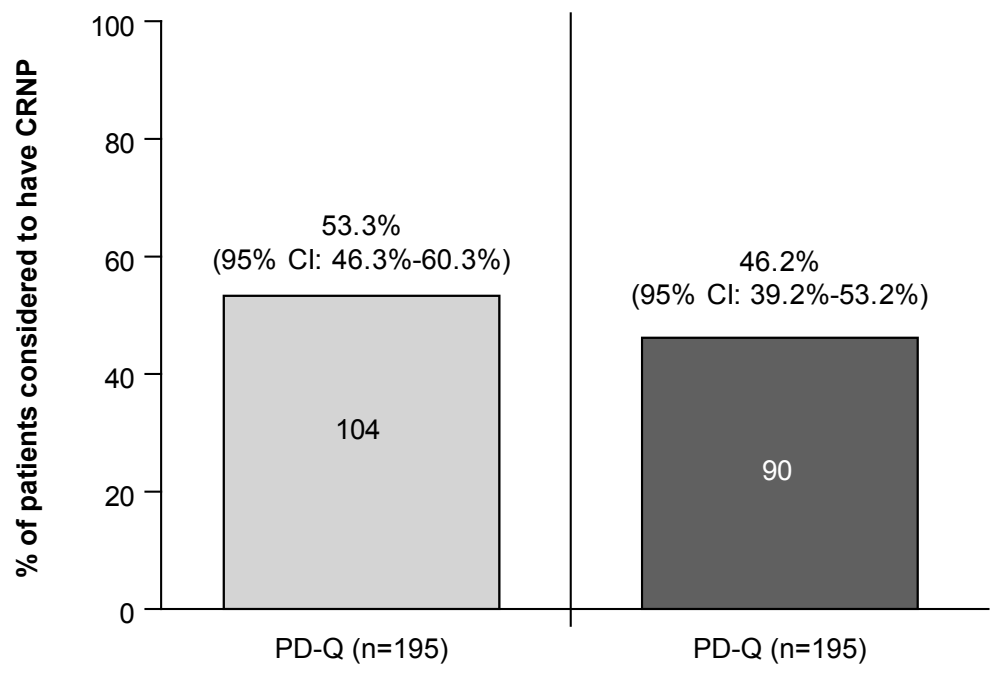

Fig (1). The percentage of patients considered to have cancer-related neuropathic pain in the opinion of the physicians, before (light grey) and after (dark grey) reviewing scores on the PainDETECT questionnaire. CRNP, cancer-related neuropathic pain; PD-Q, PainDETECT questionnaire.

surgery], adjuvant [i.e., following surgery], advanced/metastatic) on the completed CRFs was missing for $19(21.1 \%)$ CRNP patients. Of the 47 patients with completed CRFs and for whom the type of treatment was recorded, 4 had received adjuvant cancer treatment, 16 had received advanced or metastatic treatment, 5 had received neoadjuvant treatment, and 25 had no recorded treatment for cancer. A single patient could have more than one treatment type recorded.

Data on surgical procedures (i.e., non-drug treatment of cancer) were missing for the 49 of 90 patients (54.4\%). For the patients for whom these data were collected, the surgical procedures were recorded verbatim and therefore not readily categorised. Among the most commonly recorded surgical procedures were excision, mastectomy, bilateral salpingooophorectomy, and abdominal hysterectomy.

\section{Health-Related QoL in CRNP Patients}

\section{EQ-5D Health Questionnaire (EuroQoL) [20]}

Patients rated their own perception of their health (how good or bad) on a visual analogue scale (VAS) $(0=$ worst imaginable health state to $100=$ best imaginable health state). The mean health state score for the CRNP population was 53.2 (95\% CI: 47.5-59.0). In addition, patients evaluated five dimensions of health-related QoL (mobility, self-care, usual activities, pain/discomfort, and anxiety/depression) on a 3-point response scale (no problems, some problems, and extreme problems/inability). Responses to these items were 
combined into one utility score on a scale of 0 to 1 . Higher scores indicated better health. The mean utility score for CRNP patients was 0.55 (95\% CI: 0.49-0.61). Data were missing for 26 of 90 patients.

Table 2. Therapeutic Management of Neuropathic Pain in the CRNP Population

\begin{tabular}{|c|c|c|}
\hline $\begin{array}{c}\text { Prescribed Treatment for } \\
\text { Neuropathic Pain, } \\
\mathbf{n}(\mathbf{\%})\end{array}$ & $\begin{array}{c}\text { Previous } \\
\mathbf{n}=\mathbf{9 0}\end{array}$ & $\begin{array}{c}\text { Current } \\
\mathbf{n = 9 0}\end{array}$ \\
\hline \hline Non-opioid analgesics & $51(56.7)$ & $25(27.8)$ \\
\hline Weak opioid & $22(24.4)$ & $9(10.0)$ \\
\hline Strong opioid & $36(40.0)$ & $21(23.3)$ \\
\hline Antidepressants & $14(15.6)$ & $7(7.8)$ \\
\hline Anticonvulsants & $17(18.9)$ & $12(13.3)$ \\
\hline Muscle relaxants & $2(2.2)$ & $1(1.1)$ \\
\hline Corticosteroids & $3(3.3)$ & $2(2.2)$ \\
\hline Antispasmodics & 0 & 0 \\
\hline Anxiolytics & $3(3.3)$ & $1(1.1)$ \\
\hline Other & $7(7.8)$ & $6(6.7)$ \\
\hline None & 0 & $11(12.2)$ \\
\hline
\end{tabular}

${ }^{a}$ More than 1 treatment type was possible for each patient.

${ }^{\mathrm{b}}$ Examples of drugs included in each treatment group: non-opioid analgesics (e.g., paracetamol, ibuprofen), weak opioid (e.g., tramadol), strong opioid (e.g., morphine, oxycodone), antidepressants (e.g., amitriptyline, duloxetine), anticonvulsants (e.g., pregabalin, gabapentin, carbamazepine), muscle relaxants (e.g., tetrazepam, mephensin), corticosteroids (e.g., dexamethasone, prednisone), antispasmodics (e.g., baclofen, dantrolene), and anxiolytics (e.g., diazepam, alprazolam, lorazepam). CRNP, cancer-related neuropathic pain.

\section{The Sheehan Disability Scale (SDS) [21]}

The SDS assesses functional impairment in three domains (work/school, social life, and family life) using an 11 point $\operatorname{VAS}(0=$ no impairment to $10=$ extreme impairment $)$.
These scores were combined to indicate a global functional impairment score of 0 to 30 . Higher scores on the SDS indicated greater impairment. The mean total score for the CRNP population was 20.1 (95\% CI: 16.9-23.4), although there were missing data for 56 of 90 patients. Scores ranged from 1 to 30 .

Patients also indicated the number of unproductive days and the number of days lost over the past week on the SDS scale. On average, patients reported 3.7 days lost (95\% CI: 2.7-4.8) and 2.7 days unproductive (95\% CI: $1.7-3.8)$ over the past week.

\section{Patient Assessment of Disease}

During the 4-week period before the clinic visit, 52 of the 90-patient CRNP population (57.8\%) visited the doctor (reasons unknown), including nine patients who recorded four or more visits. Of 90 patients, $14(15.6 \%)$ recorded no visits. Data were missing for 24 patients $(26.7 \%)$.

All 90 CRNP patients had used some treatment over the past 4 weeks. Fifty-four patients $(60.0 \%)$ used prescription medications, and 13 (14.4\%) used non-prescription medications. The other patients used other treatments, including physiotherapy and massage.

Of the 90 CRNP patients, 26 (28.9\%) indicated that their symptoms had had an effect on their employment status, while $39(43.3 \%)$ indicated that it had not. Employment status data were missing for $27.8 \%$ of patients.

\section{Evaluation of PainDETECT Screening Tool [15]}

Six of the 11 surveyed physician population $(54.5 \%)$ responded "yes" to the question "Did you find the PainDetect Questionnaire useful?" and three $(27.3 \%)$ responded "no". Responses to this question were missing for two physicians.

Physicians responded "yes" to the question "Did the PainDETECT Questionnaire help you evaluate if you think the patient has CRNP?" in relation to 69 of 195 (35.4\%) patients. They responded "no" in relation to $123(63.1 \%)$ patients, and data were missing for three patients. While no physicians indicated that they would use the PainDETECT

Table 3. Patients' Assessment of Pain - PainDETECT Preliminary Questions (0 = No Pain; $10=$ Maximum Pain)

\begin{tabular}{|c|c|c|c|}
\hline Question & & $\begin{array}{c}\text { CRNP patients } \\
\quad \mathrm{n}=90\end{array}$ & $\begin{array}{c}\text { All patients }^{\mathrm{a}} \\
\mathbf{n}=195\end{array}$ \\
\hline \multirow[t]{3}{*}{ How would you assess your pain now, at this moment? } & Mean (SD) & $4.2(2.6)$ & $3.4(2.7)$ \\
\hline & $95 \% \mathrm{CI}$ & $3.6-4.7$ & $3.1-3.8$ \\
\hline & Missing data, $\mathrm{n}$ & 1 & 2 \\
\hline \multirow{2}{*}{ How strong was the strongest pain during the past 4 weeks? } & $95 \% \mathrm{CI}$ & 7.7-8.5 & $7.0-7.7$ \\
\hline & Missing data, $\mathrm{n}$ & 1 & 1 \\
\hline \multirow[t]{2}{*}{ How strong was the pain during the past 4 weeks, on average? } & Mean (SD) & $5.8(2.2)$ & $5.1(2.4)$ \\
\hline & $95 \% \mathrm{CI}$ & $5.3-6.2$ & 4.8-5.5 \\
\hline
\end{tabular}

aThe "all patient" group included the cancer-related neuropathic pain population.

CRNP, cancer-related neuropathic pain. 
screening tool for all of their patients in the future, two of 11 $(18.2 \%)$ indicated that they would use it for most patients, and six $(54.5 \%)$ indicated that they would use it for some patients. None of the physicians indicated that they would use it for only a few patients. Two physicians (18.2\%) indicated that they would not use it. Data were missing for one physician.

Table 4. Summary of Characteristics and Type of Cancer in the CRNP Population

\begin{tabular}{|c|c|}
\hline Characteristics of Cancer & $\mathrm{n}=\mathbf{9 0}$ \\
\hline \multicolumn{2}{|l|}{ Cancer type, $\mathrm{n}(\%)^{\mathrm{a}}$} \\
\hline Breast & $20(22.2)$ \\
\hline Prostate & $6(6.7)$ \\
\hline Colorectal & $5(5.6)$ \\
\hline Lung & $3(3.3)$ \\
\hline Other $^{\mathrm{b}}$ & $33(36.7)$ \\
\hline Missing data & $25(27.8)$ \\
\hline \multicolumn{2}{|c|}{ Total number of sites of metastasis, n (\%) } \\
\hline 0 & $8(8.9)$ \\
\hline 1 & $22(24.4)$ \\
\hline 2 & $11(12.2)$ \\
\hline 3 & $5(5.6)$ \\
\hline $4+$ & $2(2.2)$ \\
\hline Missing data & $42(46.7)$ \\
\hline \multicolumn{2}{|l|}{ Sites of metastasis, n (\%) } \\
\hline Bone & $22(24.4)$ \\
\hline Lymph node & $17(18.9)$ \\
\hline Lung & $11(12.2)$ \\
\hline Liver & $10(11.1)$ \\
\hline Brain & $2(2.2)$ \\
\hline Other & $17(18.9)$ \\
\hline Missing data & $40(44.4)$ \\
\hline
\end{tabular}

${ }^{a}$ A single patient could have $>1$ primary diagnosis.

bother cancer types included cervical, endometrial, sarcoma, uterine, and vulval cancer, and other MedDRA lowest-level term types of cancer, including unknown primary cancer.

CRNP, cancer-related neuropathic pain.

\section{Impact of PainDETECT End Scores on Physicians' Clini- cal Opinions}

The distribution of PainDETECT end scores in relation to the physician's clinical opinion regarding the presence (yes), or absence (no) of CRNP after consideration of the PainDETECT is shown in Table 5. Shifts in physicians' opinion before and after consideration of the PainDETECT are also shown in relation to the patient end scores (Table 5).
Table 5. Physicians' Opinion of the Number of Patients With CRNP and Shifts in Clinical Opinion After Consideration of PainDETECT, Distributed by PainDETECT End Scores

\begin{tabular}{|c|c|c|c|}
\hline & \multicolumn{3}{|c|}{ PainDETECT End Scores } \\
\hline & $\begin{array}{c}<13 \text { (unlikely) } \\
n=127\end{array}$ & $\begin{array}{c}13-18 \\
\text { (possible) } \\
\mathbf{n}=39\end{array}$ & $\begin{array}{c}>18 \\
\text { (likely) } \\
\mathrm{n}=29\end{array}$ \\
\hline \multicolumn{4}{|c|}{$\begin{array}{l}\text { Diagnosis }^{\mathrm{a}} \text { of CRNP } \\
\text { after consideration of } \\
\text { PainDETECT }\end{array}$} \\
\hline Yes & 36 & 28 & 26 \\
\hline No & 89 & 11 & 3 \\
\hline Missing & 2 & 0 & 0 \\
\hline \multicolumn{4}{|c|}{$\begin{array}{l}\text { Shifts in diagnosis }{ }^{\mathrm{a}} \text { of } \\
\text { CRNP before and after } \\
\text { consideration of } \\
\text { PainDETECT }\end{array}$} \\
\hline No to Yes & 1 & 0 & 2 \\
\hline Unknown to Yes & 1 & 1 & 1 \\
\hline Missing to Yes & 0 & 0 & 0 \\
\hline Yes to No & 16 & 3 & 0 \\
\hline Unknown to No & 7 & 0 & 0 \\
\hline Missing to No & 1 & 0 & 0 \\
\hline
\end{tabular}

a Based on physicians' response to the question "In your clinical opinion does this subject have chronic cancer related neuropathic pain?"

\section{DISCUSSION}

Before the use of the PainDETECT tool, the estimated number of outpatients with cancer considered to have CRNP in the UK sample was 104 of 195 patients experiencing chronic pain $(53.3 \%$; 95\% CI: 46.3\%-60.3\%). After physicians had an opportunity to consider the completed PainDETECT questionnaires, this estimate was slightly reduced, to $46.2 \%$ (95\% CI: 39.2\%-53.2\%). These percentage rates were higher than those based on a review of published data that estimated a prevalence of up to $39 \%$ when patients experiencing mixed pain with a neuropathic component were included [14]. They were also higher than the estimated percentage of CRNP in the full study that incorporated data from all five European countries. The estimated percentage of CRNP after considering PainDETECT scores in the full study was $32.6 \%$ (95\% CI: $29.6 \%-35.6 \%)$. The reason for the higher estimation in this UK sample is unclear. However, eight of the 11 physicians who completed the investigatorspecific CRF recorded that their areas of speciality were limited to two to three areas. Thus, the cancer types of the patients included in this study would have been influenced by the areas of speciality of the oncologists taking part. One physician who enrolled patients did not complete this CRF.

Based on the 2825 patients with recorded screening data, the prevalence of chronic pain in patients with cancer was estimated at $9.0 \%$. This estimation is lower than previously 
reported prevalence of chronic pain in patients with cancer. A systematic review estimated that pain (not specifically chronic pain) was experienced by $64 \%$ of patients at advanced stages and at $33 \%$ of patients undergoing cancer treatment or cancer survivors [3]. Another study estimated the prevalence of chronic pain in cancer survivors at approximately $20 \%$ [22]. The lower prevalence estimated in the current study may be owing to the strict enrollment criteria which excluded patients who experienced chronic pain for less than 3 months and/or patients whose chronic pain was effectively treated with pain medication.

The most common cancer type in this UK sample was breast cancer. This is consistent with Cancer Research UK 2009 estimates in which breast cancer was identified as the most common cancer in the UK [2]. Breast cancer accounted for $30.6 \%$ of female cancer cases in the UK in 2009 , followed by bowel cancer (11.7\%) and lung cancer (11.6\%) [2]. In men, the most commonly diagnosed cancer for the same period was prostate cancer $(25.2 \%)$, followed by lung cancer (14.2\%) and bowel cancer $(14.0 \%)$ [2]. The percentage of patients with a primary diagnosis of prostate cancer within the CRNP population reported in this article was relatively low $(6.7 \%)$, yet six of the $11(54.6 \%)$ surveyed physicians included prostate cancer among their particular areas of expertise. Consequently, the cancer types within this sample do not appear to be entirely influenced by the area of expertise of the physicians, for prostate cancer at least. On the other hand, cancer type was unrecorded for $27.8 \%$ of the CRNP population. Other areas of speciality recorded by the 11 physicians were: breast cancer (5 physicians), colorectal cancer (four physicians) lung cancer (three physicians), and "other" (seven physicians). An alternative explanation is that the recorded cancer types in this CRNP population may be indicative of the likelihood of experiencing neuropathic pain. For example, breast cancer has been associated with high levels of neuropathic pain [23, 24]. In the European Pain in Cancer (EPIC) survey, self-reported incidence of pain was highest in patients with lung and bowel cancer, and lowest in patients with prostate cancer or leukaemia [25]. However, the primary cancer diagnosis data were missing for $27.8 \%$ of patients in the current study, so these data should be interpreted with caution.

Based on statistical models of published data, there are more new cases of cancer in men in the UK (estimated 163,650 ) than in women (estimated 146,630) [26]. In the "all patient" population of outpatients with cancer experiencing chronic pain, there were more female outpatients $(n=111)$ than male $(n=81)$, although data were included from only 12 physicians in total. The demographic was similar in the CRNP population (57 women; $31 \mathrm{men}$ ). The men were also older than the women, on average. The pattern of age-group by gender typifies the most common cancers for each sex, as the greatest average number of new breast cancer cases by age in the UK during 2007-2009 was in the 25- to 49-year age category (contributing $44 \%$ of the cancer types for this age group in women) [2]. The greatest average number of new prostate cancer cases by age in the UK during 20072009 was in the 50- to 74-year age category (contributing $27 \%$ of the cancer types for this age group in men) [2]. In the CRNP sample reported here, the majority of women were aged between 45 and 64 years, while the majority of men were aged 65 years and older.
A further aim of this study was to assess the usefulness of the PainDETECT screening tool in an outpatient oncology environment based on the physicians self-reports. This questionnaire has already been validated and is used in other populations. Despite indicating that PainDETECT did not help them evaluate whether a patient had CRNP in respect of $63 \%$ of the study patients, six of the 11 surveyed physicians $(54.5 \%)$ found PainDETECT a useful tool, and eight $(72.8 \%)$ indicated that they would use it in the future with at least some of their patients. The percentage of patients considered to have CRNP after clinicians had considered the PainDETECT scores was 7\% lower than their estimate before considering the PainDETECT scores, demonstrating that physicians were influenced by the outcomes of this tool in some cases. Consideration of the data regarding this shift shows that the majority of changes in opinion occurred for patients with end scores $<13$ (indicative that underlying CRNP was unlikely). Physicians changed from a positive (yes) to a negative (no) diagnosis of CRNP regarding 16 of the 127 patients with an end score of $<13$. There were also shifts from an initial "unknown" diagnosis for seven of the patients with this end score. A greater proportion of patients with PainDETECT end scores of $<13$ received a diagnosis of neuropathic pain after examination of this questionnaire (36 of 127 [28.3\%]) than patients with PainDETECT end scores of $>18$ (indicative that underlying CRNP was likely) who were considered not to experience neuropathic pain (three of 27 [11.1\%]; Table 5). This trend is consistent with the findings of a recent review of pharmacologic treatments for CRNP that identified a shift from a trend of underdiagnosis of CRNP to hyperdiagnosis, explained by the researchers as a potential confusion between symptoms of neuropathic pain and hyperalgesia resulting from opioid administration [27]. Consequently, identifying screening tools that can help physicians characterise and diagnose the nature of pain is paramount to improving pain management in cancer patients, particularly as poor pain assessment has been identified as a contributing factor to the current undertreatment of cancer pain in general $[28,29]$.

A review of data from the European Palliative Care Research Collaborative established that the sensitivity and specificity of PainDETECT as a tool to identify neuropathic pain in patients with cancer was less reliable than that shown in patients without cancer [33]. Other tools that may help physicians to identify a neuropathic pain component include The Leeds Assessment of Neuropathic Pain Symptoms and Signs, the Northwick Park Neck Pain Questionnaire, the Neuropathic Pain Symptom Index, and the DN4 [30]. There is no evidence that these tools are any more accurate than the PainDETECT in identifying CRNP. Due to complications specific to patients with cancer (e.g., use of oncological treatment, use of strong opioids), the PainDETECT questionnaire may require some adjustments to be a more sensitive classification tool of neuropathic pain and to accurately identify CRNP [30].

Pain management in this population is complicated by the fact that many cancer patients experience mixed pain (with a neuropathic component) that may require additional prescribing strategies [14]. Insufficient physician knowledge of appropriate pharmacologic treatment of the neuropathic component of cancer pain has also been identified as a barrier to effectively treating cancer pain [31]. The most recent 
National Institute for Health and Clinical Excellence (NICE) clinical guidelines for the treatment of pain in palliative care recommend strong opioids as first-line maintenance treatment [1]. The NICE clinical guideline for the treatment of neuropathic pain recommend oral amitriptyline or pregabalin as first-line treatment [32]. There are no NICE guidelines specifically for neuropathic pain in cancer patients. The European Society for Medical Oncology guidelines recommend that neuropathic pain in cancer patients be treated with opioid and non-opioid analgesics, specifically tricyclic antidepressants (amitriptyline) or anticonvulsants (gabapentin) [33]. Radiotherapy is recommended for neuropathic pain due to bone metastases [33].

Raising awareness of effective prescribing strategies for cancer patients with CRNP, or a mixed pain condition with a neuropathic component, may also improve QoL in cancer patients, particularly as patients with CRNP have been reported to be negatively affected by their pain condition [34]. Responses to the self-reported measures on pain and QoL reported here are consistent with these reports.

Limitations to the interpretation of these data are due, in part, to missing data arising from missing or incomplete survey responses. As this was a non-interventional study, it did not incorporate monitoring or quality checking for CRF completion. The majority of physicians completed the investigator-specific CRF (11 of 12), but there were also missing or incomplete patient CRFs. The way the CRF was designed may have contributed to the lack of patients responses because every assessment in the CRF started with the checkbox "not done", this may have given the participant the wrong idea that they do not have to complete the whole questionnaires. Additionally, some of the information had to come from the patient's medical record which could have contributed to the low completion rates. Finally, some of the patient questionnaires, for example the impact on employment status and money spent on medication, may have been considered inappropriate for some patients. In addition, the outpatient sample included in the study was not necessarily representative of the outpatient population of cancer patients as a whole. The types of cancer experienced by the patients would be limited by the area of expertise/speciality of the physicians who enrolled patients for the survey. Consequently, these data should be interpreted with caution.

\section{CONCLUSION}

The prevalence of CRNP in this subpopulation of adult cancer patients attending outpatient clinics in the UK was estimated at $53.3 \%$ before physicians considered the completed PainDETECT questionnaires. The opinions of the physicians were influenced to a certain extent by the PainDETECT tool. In particular, some diagnoses were switched from a positive to a negative diagnosis of CRNP in cases where PainDETECT end scores indicated that neuropathic pain was unlikely. However, the revised estimated percentage of $46.2 \%$ remained higher than estimations found in other studies. The interpretation of these study results should be reviewed in the light of the limited data.

Pain in cancer patients is commonly undertreated. Effective management of pain is partly dependent on correctly identifying the source and type of pain (i.e., nociceptive or neuropathic). Consequently, identifying screening/diagnosis tools that help oncologists to characterise and diagnose the nature of cancer-related pain would enable them to appropriately tailor pharmacologic treatment, and, ultimately, manage pain more effectively. However, further studies are needed to assess the usefulness of the PainDETECT questionnaire within the oncology outpatient population.

\section{CONFLICT OF INTEREST}

A. Birtle and S. Davidson have no conflict of interest with this article content. G Atkinson and C. van Litsenburg are employees of Pfizer and own Pfizer stock and Pfizer stock options.

\section{ACKNOWLEDGEMENT}

This study was funded by Pfizer Ltd. Medical writing support for the development of this manuscript was provided by Brenda Meyer, PhD, of Engage Scientific Solutions and was funded by Pfizer Ltd. The authors would like to thank Sarah Wensley, Pfizer Ltd and all the UK investigators (full list reported elsewhere) [18] for their contribution to the study and Sarah Bowen for her role in the statistical analysis. The authors were not compensated for their work on the manuscript.

\section{ABBREVIATIONS}

$$
\begin{aligned}
& \mathrm{CI}=\text { Confidence interval } \\
& \mathrm{CRF}=\text { Case report form } \\
& \text { CRNP }=\text { Cancer-related neuropathic pain } \\
& \text { EuroQOL }=\text { EQ-5D Health Questionnaire } \\
& \text { m-BPI-sF }=\text { Modified Brief Pain Inventory Short Form } \\
& \text { MedDRA }=\text { Medical Dictionary for Regulatory Activities } \\
& \text { NICE }=\text { National Institute for Health and Clinical Ex- } \\
& \text { cellence } \\
& \text { QOL }=\text { Quality of life } \\
& \text { SDS } \quad=\text { Sheehan Disability Scale } \\
& \text { VAS }=\text { Visual analogue scale }
\end{aligned}
$$

\section{REFERENCES}

[1] National Institute for Health and Clinical Excellence. Opioids in palliative care: safe and effective prescribing of strong opioids for pain in palliative care of adults. NICE guidance 140. 2012. Available from: http://www.nice.org.uk/nicemedia/live/13745/ 59285/59285.pdf [Accessed January 21, 2013].

[2] Cancer Research UK. Cancer incidence for all cancers combined. 2012. Available from: http://www.cancerresearchuk.org/cancerinfo/cancerstats [Accessed November 28, 2012].

[3] van den Beuken-van Everdingen MH, de Rijke JM, Kessels AG, et al. Prevalence of pain in patients with cancer: a systematic review of the past 40 years. Ann Oncol 2007; 18(9): 1437-49.

[4] Breuer B, Fleishman SB, Cruciani RA, Portenoy RK. Medical oncologists' attitudes and practice in cancer pain management: a national survey. J Clin Oncol 2011; 29(36): 4769-75.

[5] Fairchild A. Under-treatment of cancer pain. Curr Opin Support Palliat Care 2010; 4(1): 11-5.

[6] World Health Organization. Cancer pain relief: 2nd ed. with a guide to opioid availability. 1996. Available from: http://whqlibdoc.who.int/publications/9241544821.pdf [Accessed February 1, 2012].

[7] International Association for the Study of Pain. IASP Taxonomy. 2011. Available from: http://www.iasp-pain.org/Content/Navi- 
gationMenu/GeneralResourceLinks/PainDefinitions/ [Accessed December 16, 2012].

[8] Nicholson B. Differential diagnosis: nociceptive and neuropathic pain. Am J Manag Care 2006; 12 (9 Suppl): S256-62.

[9] Huntoon MA. Cancer pain management. Minn Med 2001; 84(7): 36-9.

[10] Swarm R, Abernethy AP, Anghelescu DL, et al. Adult cancer pain. J Natl Compr Cancer Netw 2010; 8(9): 1046-86.

[11] Bhatnagar S, Mishra S, Roshni S, Gogia V, Khanna S. Neuropathic pain in cancer patients-prevalence and management in a tertiary care anesthesia-run referral clinic based in urban India. J Palliat Med 2010; 13(7): 819-24

[12] Kerba M, Wu JS, Duan Q, Hagen NA, Bennett MI. Neuropathic pain features in patients with bone metastases referred for palliative radiotherapy. J Clin Oncol 2010; 28(33): 4892-7.

[13] Cleeland CS, Farrar JT, Hausheer FH. Assessment of cancerrelated neuropathy and neuropathic pain. Oncologist 2010; 15 Suppl 2: 13-8.

[14] Bennett MI, Rayment C, Hjermstad M, Aass N, Caraceni A, Kaasa S. Prevalence and aetiology of neuropathic pain in cancer patients: a systematic review. Pain 2012; 153(2): 359-65.

[15] Freynhagen R, Baron R, Gockel U, Tolle TR. painDETECT: a new screening questionnaire to identify neuropathic components in patients with back pain. Curr Med Res Opin 2006; 22(10): 191120.

[16] Amris K, Jespersen A, Bliddal H. Self-reported somatosensory symptoms of neuropathic pain in fibromyalgia and chronic widespread pain correlate with tender point count and pressure-pain thresholds. Pain 2010; 151(3): 664-9.

[17] Baron R, Tolle TR, Gockel U, Brosz M, Freynhagen R. A crosssectional cohort survey in 2100 patients with painful diabetic neuropathy and postherpetic neuralgia: Differences in demographic data and sensory symptoms. Pain 2009; 146(1-2): 34-40.

[18] Garzón Rodríguez C, Lyras L, Olay Gayoso L, et al. Cancer-related neuropathic pain in out-patient oncology clinics: a European survey. BMC Palliat Care 2013; 12(1): 41.

[19] Mendoza TR, Chen C, Brugger A, et al. The utility and validity of the modified brief pain inventory in a multiple-dose postoperative analgesic trial. Clin J Pain 2004; 20(5): 357-62.

[20] The EuroQol Group. EuroQol-a new facility for the measurement of health-related quality of life. Health Policy 1990; 16(3): 199208.

[21] Bouhassira D, Attal N, Alchaar H, et al. Comparison of pain syndromes associated with nervous or somatic lesions and development of a new neuropathic pain diagnostic questionnaire (DN4). Pain 2005; 114(1-2): 29-36.

[22] Green CR, Hart-Johnson T, Loeffler DR. Cancer-related chronic pain: examining quality of life in diverse cancer survivors. Cancer 2011; 117(9): 1994-2003.

[23] Jung BF, Herrmann D, Griggs J, et al. Neuropathic pain associated with non-surgical treatment of breast cancer. Pain 2005; 118(1-2): $10-4$

[24] Vilholm OJ, Cold S, Rasmussen L, Sindrup SH. Sensory function and pain in a population of patients treated for breast cancer. Acta Anaesthesiol Scand 2009; 53(6): 800-6.

[25] Breivik H, Cherny N, Collett B, et al. Cancer-related pain: a panEuropean survey of prevalence, treatment, and patient attitudes. Ann Oncol 2009; 20(8): 1420-33.

[26] Ferlay J, Parkin DM, Steliarova-Foucher E. Estimates of cancer incidence and mortality in Europe in 2008. Eur J Cancer 2010; 46(4): 765-81.

[27] Vadalouca A, Raptis E, Moka E, et al. Pharmacological treatment of neuropathic cancer pain: a comprehensive review of the current literature. Pain Pract 2012; 12(3): 219-51.

[28] Portenoy RK. Treatment of cancer pain. Lancet 2011; 377(9784): 2236-47.

[29] Von Roenn JH, Cleeland CS, Gonin R, Hatfield AK, Pandya KJ. et al. Physician attitudes and practice in cancer pain management. A survey from the Eastern Cooperative Oncology Group. Ann Intern Med 1993; 119(2): 121-6.

[30] Rayment C, Hjermstad MJ, Aass N, et al. Neuropathic cancer pain: Prevalence, severity, analgesics and impact from the European Palliative Care Research Collaborative-Computerised Symptom Assessment study. Palliat Med 2013; 27(8): 714-21.

[31] Christo PJ, Mazloomdoost D. Interventional pain treatments for cancer pain. Ann N Y Acad Sci 2008; 1138: 299-328.

[32] National Institute for Health and Clinical Excellence. Neuropathic pain. The pharmacological management of neuropathic pain in adults in non-specialist settings. NICE clinical guideline 96. 2010. Available from: http://guidance.nice.org.uk/CG96/NICE Guidance/pdf/English [Accessed January 21, 2013].

[33] Ripamonti CI, Bandieri E, Roila F; ESMO Guidelines Working Group. Management of cancer pain: ESMO Clinical Practice Guidelines. Ann Oncol 2011; 22 Suppl 6: vi69-77.

[34] Tofthagen CS, McMillan SC. Pain, neuropathic symptoms, and physical and mental well-being in persons with cancer. Cancer Nurs 2010; 33(6): 436-44.

\author{
Received: July 10, 2013 \\ Revised: November 20, 2013 \\ Accepted: November 22, 2013 \\ (C) Birtle et al.; Licensee Bentham Open.
}

This is an open access article licensed under the terms of the Creative Commons Attribution Non-Commercial License (http://creativecommons.org/licenses/by-nc/3.0/) which permits unrestricted, non-commercial use, distribution and reproduction in any medium, provided the work is properly cited. 